\title{
VALUE FUNCTION AND OPTIMAL CONTROL OF DIFFERENTIAL INCLUSIONS
}

\author{
BY
}

TZANKO DONCHEV and AMMARA NOSHEEN

\begin{abstract}
Optimal control system described by differential inclusion with continuous and one sided Perron right-hand side in a finite dimensional space is studied in the paper. We prove that the value function is the unique solution of a proximal Hamilton-Jacobi inequalities.
\end{abstract}

Mathematics Subject Classification 2010: 49J24, 34A60.

Key words: one sided Perron condition, value function, Hamilton-Jacobi equation.

\section{Introduction}

In the paper we study the following optimal control problem (denoted as $\left.P\left(0, x_{0}\right)\right)$ :

Minimize the continuous function $l(x(T))$ over the solutions (trajectories) of

$$
\dot{x}(t) \in F(x(t)), x(0)=x_{0}, t \in[0, T] .
$$

Here $F: \mathbb{R}^{n} \rightrightarrows \mathbb{R}^{n}, l: \mathbb{R}^{n} \rightarrow \mathbb{R}$. Denote by $V\left(0, x_{0}\right)$ the minimal value of $l(x(T))$.

Let $\bar{x}(\cdot)$ be an optimal trajectory for $P\left(0, x_{0}\right)$. If $\bar{x}(\tau)=\alpha$ for some $\tau \in[0, T]$, then due to Bellman principle $\bar{x}(\cdot)$ is an optimal trajectory also for the following problem $P(\tau, \alpha)$ (with value $V(\tau, \alpha)$ ), i.e.

Minimize $l(x(T))$ over the trajectories of

$$
\dot{x}(t) \in F(x), x(\tau)=\alpha, \tau \in[0, T], x \in \mathbb{R}^{n} .
$$


Description of the properties and behavior of the value function is one of the most important question in optimal control theory. We refer to $[2,7,14,17]$, where the value function is studied under Lipshitz conditions.

It is shown in [13] that the value function is the unique lower semicontinuous solution of the corresponding Hamilton-Jacoby equation, if $l(\cdot)$ is lower semicontinuous.

The generalized Hamilton-Jacobi equations are used in $[9,19]$ in order to describe the minimal time function as well as in $[4,11,16]$ to describe the reachable set of optimal control problems.

We refer to [15], where the value function is comprehensively studied for optimal control problems and for differential games.

The main target of the paper is to describe the value function as the unique continuous solution of proximal Hamilton-Jacobi inequalities under one sided Perron condition on the right-hand side.

This result is well known, when $F(\cdot)$ is locally Lipschitz $[7,14,15,18]$. We show that our assumptions are essentially weaker.

\section{Preliminaries}

We refer to $[1,7,8,18]$ for all concepts used in this paper. For $U \subset \mathbb{R}^{n}$ we denote $U^{c}=\mathbb{R}^{n} \backslash U$. Further $\operatorname{cl} A=\bar{A}$ is the closure of the set $A$. For closed bounded sets $A, B$ denote by $D_{H}(A, B)=\max \{\operatorname{Ex}(A, B), \operatorname{Ex}(B, A)\}$ - the Pompeiu-Hausdorff distance, where $\operatorname{dist}(a, B)=\inf _{b \in B}|a-b|$ and $\operatorname{Ex}(A, B)$ $=\sup _{a \in A} \operatorname{dist}(a, B)$. The multifunction $F(\cdot)$ from $\mathbb{R}^{n}$ into $\mathbb{R}^{n}$ is said to be continuous, when it is continuous with respect to the Pompeiu-Hausdorff metric. It is called upper semicontinuous (USC) at $x$ when for every $\varepsilon>0$ there exists $\delta>0$ such that $F(x)+\varepsilon \mathbb{B} \supset F(x+\delta \mathbb{B})$. With $\mathbb{B}$ we have denoted the open unit ball and by $\overline{\mathbb{B}}$ its closure. The multifunction $F: I \times \mathbb{R}^{n} \rightarrow \mathbb{R}^{n}$ is called almost continuous (almost USC), when for every $\varepsilon>0$ there exists a compact set $I_{\varepsilon} \subset I$ with Lebesgue measure meas $\left(I \backslash I_{\varepsilon}\right)<\varepsilon$ such that $F(\cdot, \cdot)$ restricted to $I_{\varepsilon} \times \mathbb{R}^{n}$ is continuous (USC). Given a closed set $S \subset E$ and $x \notin S$, we let $\operatorname{proj}_{S}(x)=\{s \in S:|x-s|=\operatorname{dist}(x, S)\}$ - the projection set of $x$ on $S$. Notice that $\operatorname{proj}_{S}(x) \neq \emptyset$, for every $x \in \mathbb{R}^{n}$.

For $p \in \mathbb{R}^{n}$ and bounded set $A \subset \mathbb{R}^{n}$, the support function is $\sigma(p, A)=$ $\sup _{v \in A}\langle v, p\rangle$. Let $F: \mathbb{R}^{n} \rightarrow 2^{\mathbb{R}^{n}}$ have nonempty compact values. The upper Hamiltonian of $F(\cdot)$ is $H_{F}(x, p)=\sigma(p, F(x))$, the lower Hamiltonian is $h_{F}(x, p)=\min _{v \in F(x)}\langle v, p\rangle=-\sigma(-p, F(x))$.

Notice that for any compact valued multifunction $F(\cdot)$, the set $\{y \in$ 
$\left.F(x):\langle p, y\rangle=h_{F}(x, p)\right\}$ is nonempty for every $p \in \mathbb{R}^{n}$.

Let $\mathcal{M}$ be complete metric space. The subset $A \subset \mathcal{M}$ is said to be residual (on second Baire category) if it contains a countable intersection of open dense sets. The set $B$ is on first Baire category if $\mathcal{M} \backslash B$ is residual.

The continuous function $w: \mathbb{R}^{+} \rightarrow \mathbb{R}^{+}$is said to be Perron function, when $w(0)=0, w(\cdot)$ is monotone nondecreasing and the unique solution of $\dot{r}=w(r), r(0)=0$ is $r(t) \equiv 0$.

Definition 1. The multifunction $F(\cdot)$ is said to be locally full Perron (continuous) when on every bounded set $A \subset \mathbb{R}^{n}$ there exists a Perron function $w(\cdot)$ such that $D_{H}(F(x), F(y)) \leq w(|x-y|)$, for every $x, y \in$ $A$. Similarly $F(\cdot)$ is called locally one sided Perron continuous (OSP) if $h_{F}(x, x-y)-h_{F}(y, x-y) \leq w(|x-y|)|x-y|$, for every $x, y \in A$ (here the lower Hamiltonian can be replaced by the upper one). When $w(r)=L r$, i.e. $h_{F}(x, x-y)-h_{F}(y, x-y) \leq L|x-y|^{2}$ for some $L \in \mathbb{R}$ (notice that here $L<0$ is possible) the multifunction $F(\cdot)$ is called locally one sided Lipschitz (OSL). We refer the reader to [10] for the properties of OSL multimaps.

Now we give some concepts of nonsmooth analysis used in the paper.

Let $f$ be extended real valued lower semicontinuous function. Recall that $\operatorname{dom} f:=\left\{x \in \mathbb{R}^{n}: f(x)<\infty\right\}$ and epif $:=\{(x, r) \in \operatorname{dom} f \times \mathbb{R}: r \geq$ $f(x)\}$.

Let $S \subset \mathbb{R}^{n}$ be closed. The proximal normal cone (cf. $\left.[5,7]\right)$ at $x \in S$ is $N_{S}^{P}(x):=\left\{\zeta \in \mathbb{R}^{n}: \exists \gamma>0, \eta>0 ;\langle\zeta, y-x\rangle \leqslant \gamma|y-x|^{2}, \forall y \in S \cap x+\eta \mathbb{B}\right\}$.

A vector $\xi \in \mathbb{R}^{n}$ is called a proximal subgradient of the lower semicontinuous function $f$ at $x \in \operatorname{dom} f$ if $(\xi,-1) \in N_{\text {epi } f}^{P}(x, f(x))$. The set of all proximal subgradients is denoted by $\partial_{P} f(x)$ and is called proximal subdifferential of $f$ at $x$.

Let the function $f: \mathbb{R}^{n} \rightarrow \mathbb{R}$ be upper semicontinuous. Then proximal superdifferential of $f$ at $x \in d o m f$ is defined as $\partial^{P}(f(x))=-\partial_{P}(-f(x))$.

Let $\Omega \subset \mathbb{R}^{n}$ be an open set. Denote by $\mathcal{F}(\Omega)$ the set of all lower semicontinuous functions $\varphi: \Omega \rightarrow \mathbb{R}$ which are not identically $-\infty$.

Definition 2. Suppose $\varphi \in \mathcal{F}(\Omega)$. The system $\dot{x}(t) \in F(x(t))$ is said to be $\varphi$-weakly decreasing if for any $a \in \Omega$ there exists a trajectory $x(\cdot)$ which lies in $\Omega$ with $x(0)=a$ such that the map $t \rightarrow \varphi(x(t))$ is decreasing. We will write further the system $(\varphi, F)$ is weakly decreasing. 
The system $(\varphi, F)$ is said to be $\varphi$-strongly decreasing if for every trajectory $x(\cdot)$ which lies in $\Omega$ the map $t \rightarrow \varphi(x(t))$ is decreasing. It is said to be strongly increasing if $(-\varphi, F)$ is strongly decreasing.

The following proposition can be found in [7], p. 67 .

Proposition 1. Let $f \in \mathcal{F}(\Omega)$, and $(\zeta, 0) \in N_{\text {epif }}^{P}(x, f(x))$. Then for every $\varepsilon>0$ there exists $y$ with $|x-y|<\varepsilon$ and $(\eta,-\lambda) \in N_{\text {epif }}^{P}(y, f(y))$ such that:

$$
\lambda>0, \quad|f(x)-f(y)|<\varepsilon, \quad\|(\zeta, 0)-(\eta,-\lambda)\|<\varepsilon .
$$

Consider the following differential inclusion:

$$
\dot{x}(t) \in G(t, x(t)), x(0)=x_{0}, t \in[0, T] .
$$

Here $G: \mathbb{R} \times \mathbb{R}^{n} \rightrightarrows \mathbb{R}^{n}$ is with nonempty values. In the paper we study the case when $G(t, x) \subset F(x)$ is a submultifunction of $F(\cdot)$. Although $F(\cdot)$ is autonomous, the submultifunctions depend in general on $t$.

Take a subdivision $\Delta=\left\{0<t_{1}<\cdots<t_{N}=T\right\}$. If the approximate solution $y(\cdot)$ is already defined on $\left[0, t_{k}\right]$, then we take $y(t)=y\left(t_{k}\right)+\left(t-t_{k}\right) f_{k}$ on $\left(t_{k}, t_{k+1}\right]$, where $f_{k} \in G\left(t_{k}, y\left(t_{k}\right)\right)$ is arbitrary. Let $|\Delta|=\max _{k} \mid t_{k+1}-$ $t_{k} \mid$. The absolutely continuous function $x(\cdot)$ is said to be Euler arc of the system $\dot{x} \in G(t, x), x(0)=x_{0}$ on the interval $[0, T]$ if there exists a net of subdivisions $\Delta$ with corresponding approximate solutions $y_{\Delta}(\cdot)$ such that $x(t)=\lim _{|\Delta| \rightarrow 0} y_{\Delta}(t)$. Notice that the differential inclusion (2) may have no solutions, however, the set of Euler arcs is nonempty. Clearly every Euler arc (called feasible arc) is a solution of (1).

\section{One sided Perron differential inclusions}

In this section we study OSP continuous differential inclusions.

In the sequel we will use the following assumptions:

A1: The multifunction $F(\cdot)$ is continuous with nonempty convex compact values. Furthermore there exist constants $\alpha>0$ and $\beta>0$ such that $\|F(x)\| \leq \beta+\alpha\|x\|$ (linear growth condition).

A2: $F(\cdot)$ is OSP with Perron functions $w(\cdot)$.

First we demonstrate the strength of our results in comparison with existing results in the literature. 
Given a compact set $D \subset \mathbb{R}^{n}$ and continuous set-valued mappings $F$ and $G$ acting from $\mathbb{R}^{n}$ to itself, consider the distance

$$
C_{D}(F, G):=\max _{x \in D} D_{H}(F(x), G(x))
$$

and define the following metric in the set, denoted by $C C\left(\mathbb{R}^{n}, \mathbb{R}^{n}\right)$ ), of all continuous set-valued mappings form $\mathbb{R}^{n}$ to itself with convex and compact values:

$$
\rho_{H}(F, G):=\sum_{m=1}^{\infty} \frac{C_{\mathbb{D}_{m}}(F, G)}{2^{m}\left(1+C_{\mathbb{D}_{m}}(F, G)\right)} .
$$

Here $\mathbb{D}_{m}=x_{0}+m \overline{\mathbb{B}}$. Endowed with this metric, $C C\left(\mathbb{R}^{n}, \mathbb{R}^{n}\right)$ becomes a complete metric space.

Since every continuous function is uniformly continuous on $\mathbb{D}_{m}$, it admits modulus of continuity:

$$
w_{F}^{m}(r):=\max \left\{D_{H}(F(x), F(y)):|x-y| \leq r ; x, y \in \mathbb{D}_{m}\right\} .
$$

It is shown in [9] (Theorem 3.1) that for almost all (in Baire sense) continuous multifunctions $F(\cdot)$ the function $r \rightarrow w_{F}^{m}(r)$ is full Perron for every natural $m$ and hence almost all $F \in C C\left(\mathbb{R}^{n}, \mathbb{R}^{n}\right)$ are locally full Perron. Furthermore it is shown in [9] that the set of locally OSL (and, moreover, locally Lipschitz) is on first Baire category. Furthermore, the one sided Perron condition is evidently weaker than Perron one. Notice that OSP multimaps are in general discontinuous, also $\overline{c o} F(\cdot)$ is OSP iff $F(\cdot)$ is OSP (which is not true if $F(\cdot)$ is full Perron).

We give an example of OSP differential inclusion in one dimension, which is neither full Perron, nor one sided Lipschitz.

Example 1. Let $\left\{p_{k}\right\}_{k=1}^{\infty}$ be the set of the rational numbers of the interval $[0,1]$ ordered in sequence. Define:

$$
\begin{aligned}
g(x) & =\sum_{k=1}^{\infty} \frac{1}{3^{k}}\left(\sqrt[3]{p_{k}-x}-\sqrt[3]{p_{k}+x}\right. \\
& \left.+\operatorname{sign}\left(x-p_{k}\right) \sqrt{\left|x-p_{k}\right|}+\operatorname{sign}\left(x+p_{k}\right) \sqrt{\left|p_{k}+x\right|}\right),
\end{aligned}
$$

where $\operatorname{sign}(t)$ is 1 for $t>0,-1$ for $t<0$ and 0 for $t=0$.

It is easy to see that for every constant $p$ the function $v(x)=\operatorname{sign}(x+$ p) $\sqrt{|x+p|}-\sqrt[3]{x+p}$ is OSL with a constant less than $\frac{1}{5}$. Since $\sum_{k=1}^{\infty} \frac{1}{3^{k}}=\frac{1}{2}$ one has that $g(\cdot)$ is OSL with a constant less than $\frac{1}{5}$. 
Furthermore, let $F: \mathbb{R}^{n} \rightrightarrows \mathbb{R}^{n}$ be full Perron and not OSL. For example, we can take $F(x)=[-f(|x|), f(|x|)]$, where

$$
f(x)= \begin{cases}0 & x \leq 0 \\ -x \ln (x) & 0<x \leq e^{-1} \\ x & e^{-1}<x .\end{cases}
$$

Consider the following differential inclusion

$$
\dot{x}(t) \in g(x)+F(x), x(0)=0 .
$$

Since $r \rightarrow w_{F}^{m}(r)$ is monotone non decreasing, one has that $g(\cdot)+F(\cdot)$ is OSP (cf. [3]).

However, it is not full Perron.

Further in this section we will study the system (1) for $t \in(-\infty,+\infty)$.

Proposition 2. If $A 1, A 2$ hold for every closed interval $[\tau, T]$, then the solution set of $(1)$ is nonempty compact subset of $C\left([\tau, T], \mathbb{R}^{n}\right)$ which depends continuously on the initial condition $x(\tau)=\alpha$.

Proof. The fact that the solution set is nonempty compact subset of $C\left([\tau, T], \mathbb{R}^{n}\right)$ is well known $([1,8])$. We prove the continuity of the solution set w.r.t. $\alpha$ on a fixed interval $[\tau, T]$. Let $z(\cdot)$ be a solution of $\dot{x}(t) \in$ $F(x), x(\tau)=\alpha$. We define the multifunction

$$
G(t, x)=\{v \in F(x):\langle z(t)-x, \dot{z}(t)-v\rangle \leq w(|z(t)-x|)|z(t)-x|\} .
$$

Since $y \rightarrow\langle z(t)-x, y\rangle$ is linear function, one has that $G(t, x)$ admits convex values. Fix $\varepsilon>0$, there exists a set $I_{\varepsilon} \subset[\tau, T]$ with measure greater than $T-\varepsilon$ such that $\dot{z}(\cdot)$ is continuous on $I_{\varepsilon}$. If $t_{i} \rightarrow t, x_{i} \rightarrow x$ and $g_{i} \rightarrow g$ with $g_{i} \in G\left(t_{i}, x_{i}\right)$ then it is easy to see that $\left\langle z\left(t_{i}\right)-x_{i}, \dot{z}\left(t_{i}\right)-g_{i}\right\rangle \rightarrow$ $\langle z(t)-x, \dot{z}(t)-g\rangle$. Consequently $\langle z(t)-x, \dot{z}(t)-g\rangle \leq w(|z(t)-x|)|z(t)-x|$. Thus $G(t, x) \subset F(x)$ is almost USC with nonempty convex and compact values. Thus there exists a solution $y(\cdot)$ of $\dot{x}(t) \in G(t, x(t)), x(\tau)=\beta$. Denote $r(t)=\|z(t)-y(t)\|$. One has that $\frac{d}{d t} r^{2}(t)=2\langle z(t)-y(t), \dot{z}(t)-$ $\dot{y}(t)\rangle \leq 2 w(r(t)) r(t)$. Furthermore, $r(\cdot)$ is absolutely continuous and hence a.e. differentiable. If $r(t)>0$, then $\frac{d}{d t} r^{2}(t)=2 r(t) \dot{r}(t) \leq 2 w(r(t)) r(t)$, i.e. $\dot{r}(t) \leq w(r(t)$.

Let $A=\{t: r(t)=0\}$. Recall that the point $t^{\prime} \in A$ is said to be point of (right) density when $\lim h^{-1}$ meas $\left(A \cap\left[t^{\prime}, t^{\prime}+h\right]\right)=1$. It is well known that 
$A^{\prime}$ (density points of $A$ ) has full measure. Thus the set of density points of $A$, where $r(\cdot)$ is differentiable has the same measure as $A$. Clearly in this case $\dot{r}(t)=0$, because $r(t)=0$ is constant on $A$. Consequently $r(\tau)=\left|x_{0}-y_{0}\right|$ and $\dot{r}(t) \leq w(r(t))$ for a.a. $t$. Therefore $|x(t)-y(t)| \leq r(t) \leq v(t)$, where $v(\cdot)$ is the maximal solution of $\dot{v}(t)=w(v(t)), v(\tau)=\left|x_{0}-y_{0}\right|$. The proof is complete, because $w(\cdot)$ is Perron function.

It follows from Proposition 2 and the continuity of $l(\cdot)$, that the value function is continuous, and moreover, for every bounded interval $[a, b]$ there exist constants $N$ and $M$ such that $|x(t)| \leq N$ and $|F(x(t)+\mathbb{B})| \leq M-1$, where $x(\cdot)$ is an arbitrary solution of $\dot{x} \in F(x+\mathbb{B}), x(a)=\alpha$. We will use implicitly these estimations and assume that every (approximate) solution is Lipschitz with a constant $M$.

Further we will use the following proposition:

Proposition 3 (Proposition 2.1 of [7] p. 189). Let $S \subset E$ be closed and $f(t, x) \in F(x)$, where $F(\cdot)$ satisfies A1. Suppose that for every $t \in[a, b] \subset \mathbb{R}$ and every $y \in \Omega$ there exists $s \in \operatorname{proj}_{S}(y)$ such that $\langle f(t, y), y-s\rangle \leq 0$. If $x(\cdot)$ is Euler arc of $\dot{x}(t)=f(t, x(t))$, then $\operatorname{dist}(x(t), S) \leq \operatorname{dist}(x(a), S)$, for every $t \in[a, b]$.

The following theorem is Theorem 5.7 of [7] p. 212.

Theorem 1. Under A1 the system $(\varphi, F)$ is weakly decreasing on $\Omega$ iff $h_{F}\left(x, \partial_{p} \varphi(x)\right) \leq 0$, for every $x \in \Omega$.

We need the following two lemmas to prove Theorem 2 below:

Lemma 1. Let $S$ be closed. If $(S, F)$ is weakly invariant, where $F(\cdot)$ satisfies $A 1$ and $A 2$, then there exists a selection $g(z) \in F(z)$ under which $S$ is invariant, i.e. if $x(\cdot)$ is Euler arc of $\dot{x}=g(x), x(0) \in S$ then $x(t) \in S$, for all $t \in[0, T]$.

Proof. Since $(S, F)$ is weakly invariant, one has that $h_{F}(x, p) \leq 0$, for every $p \in N_{S}^{P}(x)$ (cf. [12]).

First we define an auxiliary function $f(x)$ as follows:

We choose any $s \doteq s(x) \in \operatorname{proj}_{S}(x)$ and let $f(x)=v \in F(s(x))$ to be such that $\langle v, x-s\rangle=h_{F}(s, x-s)$ (such $v$ exists). Since $x-s \in N_{S}^{P}(s(x))$, then $\langle f(x), x-s(x)\rangle \leq 0$.

Let $y(\cdot)$ be Euler arc of $\dot{x}(t)=f(s(x(t)))$, then $y(t) \in S$ due to the definition of $f$ and Proposition 3. However, $y(t)=f(x(t))=x(t)$ for 
$y(0) \in S$, i.e. $y(\cdot)$ is a solution of $\dot{x}(t) \in F(x)$. Since $f(x) \in F(x)$, one has that the submultifunction

$$
\bar{G}(x)=\{r \in F(x):\langle s(x)-x, f(x)-r\rangle \leq w(|s(x)-x|)|s(x)-x|\}
$$

is with nonempty convex compact values due to A2. Let $g(x) \in \bar{G}(x)$ be a single valued selection, then

$$
\langle g(x), x-s\rangle=\langle s-x, f(x)-g(x)\rangle+\langle f(x), x-s\rangle \leq w(|s-x|)|s-x| .
$$

We will prove that $g(\cdot)$ is the required selection.

Denote $\operatorname{dist}(x(t), S)=m(t)$. Clearly $m(0)=0$. Using standard calculations (see eg $[7,12]$ ), one can show that

$$
m^{2}(t)-m^{2}(\tau) \leq 2 \int_{\tau}^{t} w(m(s)) m(s) d s, \text { for any } 0 \leq \tau<t \leq T .
$$

The function $s \rightarrow w(m(s)) m(s)$ is continuous. Divide the both sides of the inequality (3) on $t-\tau$ and passing to limit we derive $\frac{d}{d t} m^{2}(t) \leq$ $2 w(m(t)) m(t)$

Furthermore, $m(t)$ is absolutely continuous and hence almost everywhere differentiable. Dealing as in the proof of Proposition 2 one can show that $x(t) \in S$ on $[0, T]$.

Lemma 2. Under $A 1$ and $A 2$, an arc $\bar{x}(\cdot)$ on $[a, b]$ is a solution to (1) (with another initial condition) if and only if there exists (not necessarily autonomous) selection $f(t, x) \in F(x)$ such that $\bar{x}$ is Euler solution on $[a, b]$ of the problem: $\dot{x}=f(t, x), x(a)=\bar{x}(a)$.

Proof. Since $F(\cdot)$ is continuous with convex compact values, then every Euler trajectory is a solution. Define $B_{a}:=\{(t, \bar{x}(t)): t \geq a\}$. Clearly $B_{a}$ is closed and weakly invariant for the trajectories of $\{1\} \times F(x)$. From Lemma 1 we have that there exists a function $(1, f(t, x)) \in\{1\} \times F(x)$ such that every Euler solution of $\dot{x}=f(t, x), x(a)=\bar{x}(a)$ satisfies $(t, x(t)) \in B_{a}$. Therefore $x(t)=\bar{x}(t)$ for $t \in[a, b]$, i.e. $\bar{x}(\cdot)$ is the unique Euler trajectory. $\square$

Theorem 2. Under $A 1$ and $A 2$ the system $(\varphi, F)$ is strongly decreasing on $\Omega$ if and only if $H_{F}(x, \xi) \leq 0$, for every $x \in \Omega$ and every $\xi \in \partial_{p} \varphi(x)$.

Proof. Suppose $(\varphi, F)$ is strongly decreasing and $\xi \in \partial_{P}(\varphi(\nu))$, where $\nu \in \Omega$. Let $v_{0} \in F(\nu)$ be arbitrary. We have to show that $\left\langle v_{0}, \xi\right\rangle \leq 0$. Let $\delta>0$ be such that $\nu+\delta \overline{\mathbb{B}} \subset \Omega$ and let

$$
\mathcal{S}:=\left\{(x, r) \in \mathbb{R}^{n} \times \mathbb{R}: x \in \nu+\delta \overline{\mathbb{B}}, \varphi(x) \leq r\right\} .
$$


It is easy to see that $\mathcal{S}$ is a closed set. Let $f(x)=v=\operatorname{proj}_{F(x)}\left(v_{0}\right)$. We claim that $f(\cdot)$ is continuous. Indeed it is enough to prove that $f(\cdot)$ has a closed graph, because it is a single valued selection of $F(\cdot)$. Let $x_{i} \rightarrow z$ and $v_{i} \rightarrow w$. Evidently $w \in \operatorname{proj}_{F(z)}\left(v_{0}\right)$, because $F(\cdot)$ is continuous with convex compact values. Thus $f(\cdot)$ is continuous. Let

$$
\tilde{G}(x)=\left\{\begin{array}{l}
(f(x), 0) \text { for } x \in \nu+\delta \mathbb{B} \\
\overline{c o}\{f(y):|y-\nu|=\delta\} \times\{0\} \text { otherwise }
\end{array}\right.
$$

Therefore $\tilde{G}(x)$ is USC with convex compact values. Since $(\mathcal{S}, \tilde{G}(x))$ is weakly invariant, one has that $\langle(f(\nu), 0),(\xi,-1)\rangle=\left\langle v_{0}, \xi\right\rangle \leq 0$.

Conversely. Let $a \in \mathbb{R}$ and let $\hat{x}(\cdot)$ be feasible arc such that $H_{F}(x$, $\left.\partial_{P} \varphi(\hat{x}(a))\right) \leq 0$. We have to show that for every $b>a$ sufficiently close to $a$ the following inequality is true $\varphi(\hat{x}(a)) \geq \varphi(\hat{x}(b))$. To this end we let $S=\overline{\{(x, r): x \in \Omega, r \geq \varphi(x)\}}$ and pick $M>0$ such that $\hat{x}(a)+4 M \overline{\mathbb{B}} \subset$ $\Omega$ and $\|\hat{x}(t)-\hat{x}(a)\|<M$ for every $t \in[a, b]$. Consequently $(x, r) \in$ $(\hat{x}(a)+M \overline{\mathbb{B}}) \times(\varphi(\hat{x}(a))+[-M, M])$ and $\left|\left(x^{\prime}, r^{\prime}\right)-(\hat{x}(a), \varphi(\hat{x}(a)))\right|<4 M$, for every $\left(x^{\prime}, r^{\prime}\right) \in \operatorname{proj}_{S}(x, r)$.

Due to Lemma 2 there exists a selection $f(t, x) \in F(x)$ whose unique Euler arc starting at $(a, \hat{x}(a))$ is $\bar{x}$. If $(x, r) \in(\hat{x}(a)+M \overline{\mathbb{B}}) \times(\varphi(\hat{x}(a))+M \overline{\mathbb{B}})$ then $(\eta, \lambda):=\left(x-x^{\prime}, r-r^{\prime}\right) \in N_{S}^{P}\left(x^{\prime}, r^{\prime}\right)$ and hence $(\eta, \lambda) \in N_{e p i \varphi}^{P}\left(x^{\prime}, r^{\prime}\right)$. If $\lambda<0$ then $-\frac{\eta}{\lambda} \in \partial_{P} \varphi\left(x^{\prime}\right)$. Therefore $\left\langle f\left(x^{\prime}\right),-\frac{\eta}{\lambda}\right\rangle \leq \lambda$, when $\left\langle f\left(x^{\prime}\right), \eta\right\rangle \leq$ 0 . If $\lambda=0$ then $(\eta, 0) \in N_{\text {epi }}^{P}(\hat{x}(a), \varphi(\hat{x}(a))$. Due to Proposition 1 there exist $x_{i} \rightarrow x^{\prime}, \varphi\left(x_{i}\right) \rightarrow \varphi\left(x^{\prime}\right), \varepsilon_{i} \rightarrow 0^{+}$and $\eta_{i} \rightarrow \eta$ such that $\left(\eta_{i},-\varepsilon_{i}\right) \in N_{S}^{P}\left(x_{i}, r_{i}\right)$. Since $F(\cdot)$ is continuous there exists $v_{i} \in F\left(x_{i}\right)$ with $v_{i} \rightarrow f\left(x^{\prime}\right)$. Furthermore, $\left\langle\eta_{i}, v_{i}\right\rangle \leq 0$ and hence passing to limit $\left\langle\eta, f\left(x^{\prime}\right)\right\rangle \leq 0$. We apply Proposition 3 to $S$ with $(x, r) \rightarrow(f(x), 0)$. Since the unique Euler arc starting at $(\hat{x}(a), \varphi(\hat{x}(a)))$ is $(\hat{x}(\cdot), \varphi(\hat{x}(\cdot)))$, we have $\operatorname{dist}[(\hat{x}(t), \varphi(\hat{x}(a))), S] \leq \operatorname{dist}[(\hat{x}(a), \varphi(\hat{x}(a))), S]=0$, for every $t \in[a, b]$. Therefore $\varphi(\hat{x}(b)) \leq \varphi(\hat{x}(a))$ due to the definition of $S$.

Similar result to Theorem 2 is proved in [6] when $F(\cdot)$ is Lipschitz.

Proposition 4. Under $A 1$ and $A 2$ the system $(\varphi, F)$ is strongly increasing on $\Omega$ iff $h_{F}(x, \xi) \geq 0, \forall \xi \in \partial^{P} \varphi(x)$ and $\forall x \in \Omega$.

Proof. The system $(\varphi, F)$ is strongly increasing on $\Omega$ iff the system $(-\varphi, F)$ is strongly decreasing on $\Omega$. It follows from Theorem 2 that $H_{F}(x, \xi) \leq 0, \forall \xi \in \partial_{P}(-\varphi(x))$. The latter is clearly equivalent to $-H_{F}(x$, $-\xi) \geq 0$, for every $\xi \in-\partial_{P}(-\varphi(x))=\partial^{P}(\varphi(x))$. However, $-H_{F}(x,-\xi)=$ $h_{F}(x, \xi)$, i.e. $h_{F}(x, \xi) \geq 0$, for every $\xi \in \partial^{P}(\varphi(x))$. 


\section{Value function}

In this section we prove the main results of the paper.

Let $\varphi: \mathbb{R} \times \Omega \rightarrow \mathbb{R}$ be continuous. Clearly then Theorem 1 and Proposition 4 can be rewritten as:

Proposition 5. a) Under A1 the system $(\varphi, F)$ is weakly decreasing iff $\vartheta+h_{F}(x, \xi) \leq 0, \forall(\vartheta, \xi) \in \partial_{P} \varphi(t, x), \forall(t, x) \in \mathbb{R} \times \Omega$.

b) Under $A 1, A 2$ the system $(\varphi, F)$ is strongly increasing iff $\vartheta+h_{F}(x, \xi) \geq$ $0, \forall(\vartheta, \xi) \in \partial^{P} \varphi(t, x), \forall(t, x) \in \mathbb{R} \times \mathbb{R}^{n}$.

Proposition 6. Suppose $A 1$ and $A 2$ are satisfied. Let $\bar{x}(\cdot)$ be feasible trajectory, i.e. $x(\cdot)$ is a solution of $(1)$.

If there exists a continuous function $\varphi(x)$ on $\mathbb{R}^{n}$, satisfying

$$
\begin{aligned}
& \vartheta+h_{F}(x, \xi) \geq 0, \forall(\vartheta, \xi) \in \partial^{P} \varphi(t, x), \forall(t, x) \in \mathbb{R} \times \mathbb{R}^{n} \\
& \varphi(T, x)=l(x), \varphi(\tau, \alpha)=l(\bar{x}(T)) .
\end{aligned}
$$

then $\bar{x}$ solves $P(\tau, \alpha)$ and the value of the problem $P(\tau, \alpha)$ is $\varphi(\tau, \alpha)$.

Proof. Due to Proposition 4 , the system $(\varphi, F)$ is strongly increasing. Then $\varphi(x(0))=l(\bar{x}(T))$ the value of the problem $P\left(x_{0}\right)$. Furthermore $\bar{x}(\cdot)$ is its solution, because the minima is attained at $l(\bar{x}(T))$.

Theorem 3. Under $A 1$ and $A 2$ there is unique continuous function $\varphi:(-\infty, T] \times \mathbb{R}^{n} \rightarrow \mathbb{R}$ satisfying:

$$
\begin{aligned}
& \vartheta+h_{F}(x, \xi) \geq 0, \forall(\vartheta, \xi) \in \partial^{P} \varphi(t, x) \text { and } \forall(t, x) \in(0, T) \times \mathbb{R}^{n}, \\
& \vartheta+h_{F}(x, \xi) \leq 0, \forall(\vartheta, \xi) \in \partial_{P} \varphi(t, x) \text { and } \forall(t, x) \in(0, T) \times \mathbb{R}^{n}, \\
& l(x)=\varphi(T, x), \forall x \in \mathbb{R}^{n} .
\end{aligned}
$$

This function $\varphi(\cdot)$ is the value function $V(\cdot)$.

Proof. From Proposition 6 we know that $V(\cdot)$ satisfies $\vartheta+h_{F}(x, \xi) \geq$ $0, \forall(\vartheta, \xi) \in \partial^{P} \varphi(x)$ and $\forall(t, x) \in(0, T) \times \mathbb{R}^{n}, \varphi(T, x)=l(x), \forall x \in \mathbb{R}^{n}$. It remains to show that $\vartheta+h_{F}(x, \xi) \leq 0, \forall(\vartheta, \xi) \in \partial_{P} \varphi(t, x)$ and $\forall(t, x) \in$ $(-\infty, T] \times \mathbb{R}^{n}$.

When $V(\tau, \alpha)$ is finite, there exists an optimal arc $\bar{x}$ for $P(\tau, \alpha)$ and along $\bar{x}, V(\cdot)$ is constant, i.e. $t \rightarrow V(t, \bar{x}(t))$ is constant on $[\tau, T]$. Thus the system $(V, F)$ is weakly decreasing. By virtue of Proposition 5 we derive $\vartheta+h_{F}(x, \xi) \leq 0, \forall(\vartheta, \xi) \in \partial_{P} \varphi(t, x)$. 
Let $\psi$ be another function, satisfying proximal Hamilton-Jacobi inequalities.

We know that $(\psi, F)$ is weakly decreasing and hence $\psi(t, x(t)) \leq \psi(\tau, x(\tau))$ when $t \geq \tau$. Letting $t \uparrow T$ we deduce $l(x(T))=\psi(T, x(T)) \leq \psi(\tau, x(\tau)) \Rightarrow$ $V(\tau, x(\tau)) \leq \psi(\tau, x(\tau))$, i.e $V \leq \psi$.

Let $(\tau, \alpha)$ be any point with $\tau<T$. Thus there exists an optimal trajectory $\bar{x}$ for $P(\tau, \alpha)$.

Due to Proposition $4(\psi, F)$ is strongly increasing. Thus $\psi(T, \bar{x}(T)) \geq$ $\psi(\tau, \alpha)$. Furthermore $\psi(T, \bar{x}(T))=l(\bar{x}(T))=\psi(\tau, x) \Rightarrow V(\tau, \alpha) \geq \psi(\tau, \alpha)$, i.e. $V \geq \psi$ and hence $V(\tau, \alpha)=\psi(\tau, \alpha)$.

Remark 1. Theorem 3 implies that the value function is the unique viscosity solution of the proximal Hamilton-Jacobi equation

$$
\begin{aligned}
& \vartheta+h_{F}(x, \xi)=0, \varphi(T, x)=l(x), \quad \forall(\vartheta, \xi) \in \partial^{P} \varphi(t, x) \\
& \quad \text { and } \forall(t, x) \in(0, T) \times \mathbb{R}^{n} .
\end{aligned}
$$

Clearly all the results hold true if one replace full OSP condition on $F(\cdot)$ to locally OSP one.

As it was pointed out $C C\left(\mathbb{R}^{n}, \mathbb{R}^{n}\right)$ is a complete metric space w.r.t. $\rho_{H}(\cdot, \cdot)$. Denote by $\mathfrak{C}=\left\{R \in C C\left(\mathbb{R}^{n}, \mathbb{R}^{n}\right): A 1\right.$ holds $\}$. Since $\mathfrak{C}$ is a closed subset of $C C\left(\mathbb{R}^{n}, \mathbb{R}^{n}\right)$, one has that it is also a complete metric space and almost every $G \in \mathfrak{C}$ is locally full Perron and almost every $R \in \mathfrak{C}$ is not locally OSL.

As a corollary of Theorems 3 we obtain the following result:

Theorem 4. Under A1 there exists a residual subset $\mathcal{B}$ of $\mathfrak{C}$ such that for every $\mathbf{F} \in \mathcal{B}$ the value function $V(\cdot)$ is the unique viscosity solution of the proximal Hamilton-Jacobi equation (6).

Proof. As we have seen almost every (in Baire sense) $\mathbf{F} \in \mathfrak{C}$ is locally full Perron w.r.t. $\rho_{H}(\cdot, \cdot)$. Since our results are true for every locally OSP and clearly also for locally full Perron, the proof is therefore complete.

\section{Conclusion}

We prove that the value function of optimal control problem governed by differential inclusion is the unique viscosity solution of the proximal Hamilton-Jacobi equation (6). 
The commonly used Lipschitz condition in the literature is essentially relaxed to one sided Perron one. It follows from the results of $[9,11]$ that the derived Hamilton Jacobi proximal equation holds for almost all (in Baire sense) continuous multifunctions from $\mathbb{R}^{n} \rightrightarrows \mathbb{R}^{n}$. We assume that $F(\cdot)$ is globally OSP, however, the results can be proved when $F(\cdot)$ is only locally OSP with obvious changes of the proofs.

Acknowledgements. We want to thank the anonymous referees for their valuable comments which help us to improve the paper.

The first author is supported by a grant of the Romanian National Authority for Scientific Research, CNCS-UEFISCDI, project number PNII-ID-PCE-2011-3-0154.

The second author is partially supported by Higher Education Commission, Pakistan.

\section{REFERENCES}

1. Aubin, J.-P.; Frankowska, H. - Set-Valued Analysis, Systems \& Control: Foundations \& Applications, 2, Birkhäuser Boston, Inc., Boston, MA, 1990.

2. Baumeister, J.; Leitão, A.; Silva, G.N. - On the value function for nonautonomous optimal control problems with infinite horizon, Systems Control Lett., 56 (2007), 188-196.

3. CÂRJĂ, O.; LAzU, A.I. - Lower semi-continuity of the solution set for semilinear differential inclusions, J. Math. Anal. Appl., 385 (2012), 865-873.

4. Clarke, F.H. - A proximal characterization of the reachable set, Systems Control Lett., 27 (1996), 195-197.

5. Clarke, F.H.; Ledyaev, Yu.S.; Radulescu, M.L. - Approximate invariance and differential inclusions in Hilbert spaces, J. Dynam. Control Systems, 3 (1997), 493518.

6. Clarke, F.H.; Ledyaev, Yu.S.; Stern, R.J. - Invariance, monotonicity, and applications, Nonlinear analysis, differential equations and control (Montreal, QC, 1998), 207-305, NATO Sci. Ser. C Math. Phys. Sci., 528, Kluwer Acad. Publ., Dordrecht, 1999.

7. Clarke, F.H.; Ledyaev, Yu.S.; Stern, R.J.; Wolenski, P.R. - Nonsmooth Analysis and Control Theory, Graduate Texts in Mathematics, 178, Springer-Verlag, New York, 1998. 
8. Deimling, K. - Multivalued Differential Equations, de Gruyter Series in Nonlinear Analysis and Applications, 1, Walter de Gruyter \& Co., Berlin, 1992.

9. Donchev, T. - Minimal time function and Hamilton-Jacobi inequalities, Int. J. Math. Stat., 6 (2010), S10, 14-24.

10. Donchev, T. - Properties of one-sided Lipschitz multivalued maps, Nonlinear Anal., 49 (2002), Ser. A: Theory Methods, 13-20.

11. Donchev, T.; Dontchev, A.L. - Extensions of Clarke's proximal characterization for reachable mappings of differential inclusions, J. Math. Anal. Appl., 348 (2008), 454-460.

12. Donchev, T.; Ríos, V.; Wolenski, P. - Strong invariance and one-sided Lipschitz multifunctions, Nonlinear Anal., 60 (2005), 849-862.

13. Frankowska, H. - Lower semicontinuous solutions of Hamilton-Jacobi-Bellman equations, SIAM J. Control Optim., 31 (1993), 257-272.

14. Frankowska, H.; Plaskacz, S.; Rzeżuchowski, T. - Measurable viability theorems and the Hamilton-Jacobi-Bellman equation, J. Differential Equations, 116 (1995), 265-305.

15. Plaskacz, S. - Value Functions in Control Systems and Differential Games: A Viability Method, Lecture Notes in Nonlinear Analysis, 5, Juliusz Schauder Center for Nonlinear Studies, Toruń, 2003.

16. Rios, V.R.; Wolenski, P.R. - Proximal characterization of the reachable set for a discontinuous differential inclusion, Geometric control and nonsmooth analysis, 270-279, Ser. Adv. Math. Appl. Sci., 76, World Sci. Publ., Hackensack, NJ, 2008.

17. Rungger M.; Starsberg O. - Continuity of value function for exit time optimal control problems of hybrid systems, 49th IEEE CDC (2010), Atlanta GA, USA, 42104215 .

18. Vinter, R. - Optimal Control, Systems \& Control: Foundations \& Applications. Birkhäuser Boston, Inc., Boston, MA, 2000.

19. Wolenski, P.R.; Zhuang, Y. - Proximal analysis and the minimal time function, SIAM J. Control Optim., 36 (1998), 1048-1072 (electronic).

Received: 12.XI.2012

Revised: 29. V.2013

Accepted: 20.VI.2013
Department of Mathematics, "Al. I. Cuza" University, Iaşi 700506, ROMANIA

tzankodd@gmail.com

Abdus Salam School of Mathematical Sciences, 68-B, New Muslim Town, Lahore,

PAKISTAN

hafiza_amara@yahoo.com 